\title{
An Introduction to Viewpoint Selection in Volume Visualization
}

\author{
Xinli Xu \\ State Key Laboratory of Virtual Reality Technology \\ and Systems, BeiHang University \\ Beijing, China \\ xuxl@vrlab.buaa.edu.cn
}

\author{
Bing He \\ State Key Laboratory of Virtual Reality Technology \\ and Systems, BeiHang University \\ Beijing, China \\ hebing@buaa.edu.cn
}

\begin{abstract}
Scientific researches are always accompanied with a large number of complex data. Thus, it is important to view and analysis these data or objects in a much more efficient way. Viewpoint selection can improve both the speed of rendering and the efficiency of data understanding, became a hot topic in research, and is applied to various research areas. Viewpoint selection considerably influences the amount of information contained in the projected images, has been extensively studied in many areas such as: volume rendering, image-based modeling, object recognition, etc. most of the studies utilizing the information theoretical framework. This paper reviews the relevant literatures on this topic by summarizing the development and application of the technology, and put forward some ideas for future development.
\end{abstract}

Keywords- viewpoint selection, volume rendering, viewpoint entropy, jensen-shannon entropy.

\section{INTRODUCTION}

Best viewpoint selection is an important task because it considerably influences the amount of information contained in the $2 \mathrm{D}$ projected images of $3 \mathrm{D}$ objects, and from a psychological point of view, the first impressions of the target volume leaves a strong impression to users, although current GPU-based systems are available for real-time rotation and translation of the given volume.

Obviously, there is a basic question underlying the viewpoint selection study and application, which is "what is a 'good' viewpoint for the scene?" Intuitively, the answers could be the most representative one, i.e. the one reveals largest amount of information for the underlying scene. This is the most acceptable concept in the all the relevant literatures [1-11].

Several viewpoint quality measures have been applied in computer graphics. Koenderink and Doorn [12, 13] introduced the idea of aspect graph to partition the viewing regions surrounding an object in 1970s. Arbel et al. [14] in 1999 used the entropy map to define a measure of ambiguity for the resulting belief distributions and use offline entropy maps to guide the on-line navigation. After Vázquez et al. [2] in 2001 introduced the Viewpoint Entropy (VE), and then, similarly methods were widely used, especially for volume rendering. For volumetric data, it can be $3 \mathrm{D}$ meshes, flow vectors or time-varying data, there are not work the same ways for the viewpoint selection methods.

The remainder of this paper is organized as follow.
Section 2 introduces some Information Theory concepts that is using for most methods. Section 3 shows the viewpoint selection methods work in different applications. Section 4 draws the conclusions and puts some comments for the future work.

\section{INFORMATION-THEORETIC CONCEPTS}

In this section, we will introduce the basic concepts, Jensen-Shannon Entropy and Viewpoint Entropy, which were the most common theories used in viewpoint selection. Although, it is not all the literatures use the concept of Viewpoint Entropy, but similar entropies were defined in same mode.

\section{A. Jensen-Shannon Entropy}

Information theory was introduced in $[15,16]$. JensenShannon Entropy is a basic concept in information theory to measure of uncertainty of a random variable.

Let $X$ be a finite set, $X$ be a random variable taking values $X$ in $X$ with distribution $p(x)=P[X=x]$. Likewise, let $y$ be a random variable taking values $y$ in $Y$. An information channel between two random variables (input $X$ and output $\mathrm{Y}$ ) is characterized by a probability transition matrix (composed of conditional probabilities) which determines the output distribution given by input.

The Jensen-Shannon entropy $H(x)$ of a random variable $\mathrm{X}$ is defined by

$$
H(x)=-\sum_{x \in \chi} p(x) \log p(x) .
$$

Here, $H(p)$ measures the average uncertainty of a random variable X. All logarithms are base 2 and entropy is expressed in bits. The convention that $0 \log 0=0$ is used. The conditional entropy is defined by

$$
H(Y \mid X)=-\sum_{x \in X} p(x) \sum_{y \in Y} p(y \mid x) \log p(y \mid x) .
$$

Here, $p(y \mid x)=\mathrm{P}[Y=y \mid X=x]$ is the conditional probability. The conditional entropy $H(Y \mid X)$ measures the average uncertainty associated with $\mathrm{Y}$ if we know the outcome of $\mathrm{X}$. In general, $H(Y \mid X) \neq H(X \mid Y)$ and $H(X) \geq H(X \mid Y) \geq 0$. 


\section{B. Viewpoint Entropy}

There are various criteria for evaluating viewpoint, In Vázquez's opinion [17], the Viewpoint Entropy (VE) is the proper criteria for viewpoint selection. They comparing many different criteria, simple ones to Fleishman's [18], which is the number of faces properly captured. Although, recently found some disadvantages with in VE.

Viewpoint Entropy first introduced by Vázquez et al. [2], can incorporate both the projected area and the number of faces, and can be understood as the amount of information captured by the viewpoint. Rigau et al. [19] have arrived to an equivalent measure when studying the visibility complexity of 2D scenes.

To define Viewpoint Entropy they use as probability distribution the relative area of the projected faces over the sphere of directions centered in the viewpoint (there are some assumptions here, see in section 3 ).

Viewpoint Entropy was defined as

$$
V E(p)=-\sum_{i=0}^{N_{f}} \frac{A_{i}}{A_{t}} \log \frac{A_{i}}{A_{t}} .
$$

Here, $N_{f}$ is the number of faces of the scene, $A_{i}$ is the projected area of face $i$ over the sphere, $A_{0}$ represents the projected area of background in open scenes, and $A_{t}$ is the total area of the sphere. In a closed scene, or if the point does not see the background, the whole sphere is covered by the projected faces and consequently $A_{0}=0$.

The maximum entropy is obtained when a certain point can see all the faces with the same relative projected area $A_{i} / A_{t}$. So, in an open scene the maximum viewpoint entropy is $\log \left(N_{f}+1\right)$, and in a closed scene it is equal to $\log N_{f}$. Thus define the best viewpoint as the one that has maximum entropy, i.e. maximum information captured.

There are other important viewpoint quality measures, Heuristic Measure (HM) [20] and Viewpoint Mutual Information (VMI) [23]. For HM, main factors are the fraction of visible surfaces with respect to the total number of surfaces, and the ratio between the projected area of the scene and the screen area. The VMI measure can be expressed as a Viewpoint Kullback-Leibler distance (VKL) $[11,15]$, is the distance between the normalized distribution of projected areas and the "ideal" projection. The minimum value 0 is obtained when the normalized distribution of projected areas is equal to the normalized distribution of actual areas. Thus, to select views of high quality means to minimize $K L_{v}$. In 2005, Sbert et al. [11] discussed the behavior of these three different viewpoint quality measures.

\section{VIEWPOINT SELECTION IN VOLUME RENDERING}

The visualization of $3 \mathrm{D}$ datasets receives increasing attention from both scientific and engineering community in recent years for it allows scientists to gain much insight into their data. However, coupled with large datasets, make the user's comprehension of the insights features of data more difficult. Actually, the early work on this topic is limited to polygonal mesh representations [21, 22], and several applications appear in Direct Volume Rendering (DVR) recently $[1,3,5,8,10,23]$, and nowadays applied to flow visualization [4].

\section{A. Assumptions}

To address the problem clearly, and render the scene properly, most of the viewpoint selection methods sheared the common assumption like this below [2-5, 7, 9, 10, 24].

a) All the viewpoints are located on the surface of a viewing sphere. At each viewpoint the user looks at the center of the sphere, where the volume is located.

b) During viewpoint selection, the viewpoint moves on the sphere, which means the distance between the viewpoint and the volume center is fixed and can be modified by user.

c) To ensure the projection of the volume from any view not fall outside the window, the viewing and projection parameters are appropriately set up.

Through these assumptions, the particular view of the scene almost only depends on the viewpoint attributes, so the image quality is almost depends on viewpoint selection.

\section{B. 3D Mesh / Shape Data}

Kamada and Kawai [21] defined a view to be optimal if it minimizes the number of degenerated faces under orthogonal projection. This method fails when comparing scenes with equal number of degenerated faces and it does not ensure that the user will see a large amount of detail, as discussed in Barral et al. [22], extended the idea to cope with perspective projection. Then they create a heuristic with some other parameters that weight both the number of faces seen from each point and the projected area. The function permits to explore the scene in real time. However, they have not been able to determine a good weighting scheme for the different factors. This causes some problems with objects containing holes, as these are not captured properly by the algorithm.

Vázquez et al. [2, 17] utilized the concept of information entropy from Information Theory to evaluate the quality of a viewpoint in 2001,2003 . The relative visibility of each face is defined as its probability, and the optimal view is found by maximizing the probability distribution using the entropy function. Vázquez et al. [25] also introduced techniques to accelerate the Viewpoint Entropy calculation for molecular models based on different OpenGL features.

Takahashi et al. [3] discussed viewpoint selection in the context of volume visualization based on VE. They decompose the volume into a set of feature Interval Volume components, and use the surface-based view point selection method suggested in $[2,17]$ to find the optimal view for each component. Then they calculate the globally optimal view by a compromise between the local optimal views of all the feature components.

Bordoloi and Shen [1] took a volume rendering approach and proposed that in a good view point, the visibility of a voxel should be proportional to the noteworthiness value of the voxel. They used three viewpoint characteristics associated with each view: view goodness, view likelihood, view stability. View goodness: tries to capture how closely 
the voxel visibilities for a given view match a user-input importance function. View likelihood: Intuitively, the view likelihood of a given view is the number of other viewpoints on the view sphere. View stability: denotes the maximal change in view that can occur when the camera position is shifted with in a small neighborhood. The noteworthiness value or the weight of the voxel can be determined by factors such as the opacity and color of the voxel. They also discussed view similarity and how to partition the view space. Chan et al. [26] extended Bordoloi's work by considering spatial relations between structures, after a user-specified segmentation has been given.

Ji et al. [6] present an improved static view selection technique to address some issues that were not previously considered, such as the perceived colors, curvatures, and opacities in the final image. They also proved that Bordoloi and Shen's method [1] may have occlusion in certain circumstances, and remedy the problem by considering additional important properties of the data, proposed an image-based view selection method.

Viola et al. [23] introduced an information-theoretic framework which is based on the Viewpoint Mutual Information (VMI) measure, which allows an automatic focus of attention on interesting objects. The user's only required (not limited to) interaction is to select an object of interest from a set of pre-segmented objects. They compared VMI and VE, and found that VMI is more robust to deal with any type of discretization or resolution of the dataset.

Miquel et al. [5] presented a unified and robust framework to deal with viewpoint selection and mesh saliency. The defined an information channel between the viewpoints and the polygons of the object an information channel between the set of viewpoints and the polygons of the object based on VMI too.

\section{Flow Data Visualization}

Flow simulation also plays an important role in many scientific and engineering disciplines such as climate modeling, turbulent combustion, and aircraft design, etc. Effective streamline visualization can be formulated as the problem of seed placement or streamline selection. With viewpoint selection, the insight would be much more impressive.

In 2012, Tao et al. [4] present an elegant solution to achieve goals in a unified information theoretic framework by constructing two interrelated information channels between a set of streamlines and a set of viewpoints. Based on the information channel from streamline to viewpoint, they define streamline information as a measure of streamline quality to guide streamline selection. And similarly, in the inverted channel from viewpoint to streamline, define viewpoint information to guide viewpoint selection for the selected streamlines. In their scenario, two view-dependent factors were used for computing the conditional probability, Mutual Information (MI) and Shape Characteristics (SC). The MI indicates how much information about streamline $S$ is revealed in its 2D projection $S_{v}$ under viewpoint $v$. The $S C$ indicates how stereoscopic the shape of streamline $S$ is reflected under viewpoint $v$. When selecting the best viewpoint, mainly consider the amount of information about the set of streamline $S$ revealed by viewpoint $V$. Use the definition of Viewpoint Information $(V I)$ by the conditional probability $p(s \mid v)$, similarly, use $p(v \mid s)$ for Streamline Information $(S I)$. It is the first work that applies dual information channels to solve flow visualization problems.

\section{Time-Varying Data}

As a time-varying dataset is usually large in size and time-consuming to render, to ensure that the large scale timevarying dataset can be explored in an efficient and effective way, the process of view selection should be done automatically as much as possible.

In [1], Bordoloi and Shen considered the problem of finding a good viewpoint for time-varying dataset. However, their method is to find a static view point throughout the animation so that the user can perceive the maximum summation of conditional entropy from the time series. The conditional entropy is the relative entropy of a data step based on its previous step. Compared with the method, Ji et al. [6] trying to maximize the information perceived from the time-varying data while following the view movement constraints, then finding a dynamic viewing path.

Ji et al. [6] also shows that search space for time-varying data is exponentially large. It is impractical to try all these paths and find the optimal one. By employ the dynamic programming approach, they made the whole process only takes a polynomial time complexity, which can finish all the computation in $O\left(N^{2}\right)$ time.

\section{E. $\quad N$ Views \& Visual search}

There seems to be two different ways to obtain the whole scene, one is selecting $\mathrm{N}$ view to cover the whole scene, the other is moving the camera surround the scene. The $\mathrm{N}$ Views problem were addressed to be selecting a minimal set of $\mathrm{N}$ views to representing the whole scene, it is related to art gallery problem, which is well-known NP problem [27]. The other one were treated as camera path planning [4, 28], visual search problem [29-31], these techniques are generally based on the movement of a camera around or inside the world to explore. Both topics were thoroughly investigated, and some were based on viewpoint selection [2, 30-32].

Zheng et al. [7] proposed a viewpoint suggestion pipeline that is based on feature-clustering in high-dimensional space. They use a set-cover optimization algorithm to provide a minimal set of views needed to observe all features, which can be obtained via k-means to detect important salient composite features by using gradient/normal variation as a metric to identify interesting local events.

Miquel et al. [5] shows an applicable framework to any set of viewpoints in a closed scene, considered only the geometric properties of an object, other aspects such as lighting could be incorporated. Sbert [11] discussed the N View problem too, present a new viewpoint selection algorithm based on the Kullback-Leibler distance to find the minimum representative set of views. 
Andujar et al. [28] proposed a camera path planning method for walkthrough of complex scene models. Their method is based on identifying the free-space structure of the scene and an entropy-based measurement of the relevance of a viewpoint. Sokolov et al. [33] presented a different exploration algorithms guided by Viewpoint Entropy and the total curvature of a visible surface, respectively. Barral et al. [22] presented a method for automatic exploration of static scenes. In their method, the quality of a view is computed by defining a new importance function that depends on the visible pixels of each polygon. In [30] and [31], the authors proposed two methods of virtual world exploration: an outside and an indoor exploration method. The two methods are based on the notion of Viewpoint Entropy.

\section{F. Other Applications}

Viewpoint selection measures have many potential applications. Here, we will review briefly some of them, referring the reader to the appropriate bibliography.

Image-Based Modeling and Rendering. Viewpoint selection can be use to other research areas, guiding user to minimize the overall reconstruction error in Image-Based Rendering, and helping to get the minimal set of viewpoints representing the scene.[1]

Ray Tracing. Ray tracing is an important method in Computer Graphics, it can render a high quality image, but cost a lot of rays through each pixel. However, not all pixels need this amount of supersampling. Viewpoint selection can give a measure [2], that a homogeneous region will need fewer rays than a region with geometrical discontinuities and/or high illumination gradients.

\section{G. Optimization}

During the last few years, researchers explored artificial intelligent (AI) techniques in visualization to accelerate the computing efficiency. Wang et al. [10] first introduces AI techniques into the viewpoint selection.

Wang et al. [10] reformulated the search for the viewpoint as a global optimization problem, integrating intelligent algorithms into viewpoint selection. The view directions are encoded as the particles in the Particle Swarm Optimizer (PSO), and the optimal viewpoint is generated by the iterations. The PSO iteration eliminates reluctant viewpoint evaluations, and thus improves the efficiency of the searching process.

Zhang et al. [8] also used the Artificial Intelligence algorithms, they use a new method based on shuffled frog leaping algorithm (SFLA). The SFLA is a member in the family of intelligent algorithms [34]. It is a memetic metaheuristic that is designed to seek a global optimal solution by performing an informed heuristic search using a heuristic function. They encode the candidate viewpoints as the frogs.

\section{CONCLUSIONS}

We have survey recent developments in the area of Viewpoint Selection. In our opinions, Viewpoint Selection works as an effective tool to improve the viewing impact. It can help us to view the insights, find the interested regions, and cover the saliency features of an object.

Although information based approaches applied to various applications, we anticipate more applications and wider usage of information-theoretic approaches to evaluate the viewpoint. In some applications, predefinition work is still too much, and most of them are not so easy for naïve users.

And the optimal work in this field have not been done enough, in-depth investigation since many other good intelligent algorithms have not yet been adopted, thus the consequent research would possess great potentialities and promotion space.

\section{REFERENCES}

[1] U.D. Bordoloi, and H.W. Shen, View selection for volume rendering, Visualization, 2005. VIS 05. IEEE, 2005, pp. 487-494.

[2] P. Vázquez, M. Feixas, M. Sbert, and W. Heidrich, Viewpoint Selection using Viewpoint Entropy, VMV '01, Aka GmbH, 2001, pp. 273-280.

[3] S. Takahashi, I. Fujishiro, Y. Takeshima, and T. Nishita, A featuredriven approach to locating optimal viewpoints for volume visualization, Visualization, 2005. VIS 05. IEEE, 2005, pp. 495-502.

[4] J. Tao, J. Ma, C. Wang, and C. Shene, A Unified Approach to Streamline Selection and Viewpoint Selection for 3D Flow Visualization, Visualization and Computer Graphics, IEEE Transactions on, vol. PP, no. 99, 2012, pp. 1.

[5] M. Feixas, M. Sbert, and F. Gonz A Lez, A unified informationtheoretic framework for viewpoint selection and mesh saliency, ACM Trans. Applied Perception (TAP), vol. 6, no. 1, 2009, pp. 1.

[6] G. Ji, and H. Shen, Dynamic View Selection for Time-Varying Volumes, Ieee Transactions On Visualization And Computer Graphics, vol. 12, no. 5, 2006, pp. 1109-1116.

[7] Z. Ziyi, N. Ahmed, and K. Mueller, iView: A Feature Clustering Framework for Suggesting Informative Views in Volume Visualization, Visualization and Computer Graphics, IEEE Transactions on, vol. 17, no. 12, 2011, pp. 1959-1968.

[8] Z. Yousai, and W. Bin, Optimal viewpoint selection for volume rendering based on shuffled frog leaping algorithm, Progress in Informatics and Computing (PIC), 2010 IEEE International Conference on, 2010, pp. 706-709.

[9] P. Vázquez, M. Feixas, M. Sbert, and A. Llobet, Viewpoint Entropy: A New Tool for Obtaining Good Views of Molecules, Eurographics Association, 2002, pp. 183-188.

[10] W. Yanni, Z. Dibin, Z. Yao, W. Kangjian, and Y. Tingjun, Viewpoint Selection Using PSO Algorithms for Volume Rendering, Computer and Computational Sciences, 2007. IMSCCS 2007. Second International Multi-Symposiums on, 2007, pp. 286-291.

[11] M. Sbert, D. Plemenos, M. Feixas, and F. Gonzalez, Viewpoint Quality: Measures and Applications, Eurographics Association, 2005, pp. 185-192.

[12] J.J. Koenderink, and A.J. Doorn, The internal representation of solid shape with respect to vision, vol. 32, no. 4, 1979, pp. 211-216.

[13] J.J. Koenderink, and A.J. Doorn, The singularities of the visual mapping, Bioogical Cybernetics, vol. 24, 1976, pp. 51-59.

[14] T. Arbel, and F.P. Ferrie, Viewpoint selection by navigation through entropy maps, Computer Vision, 1999. The Proceedings of the Seventh IEEE International Conference on, 1999, pp. 248-254.

[15] K.P. Murphy, Elements of Information Theory, Wiley, 1991.

[16] R.E. Blahut, Principles and practice of information theory, AddisonWesley Longman Publishing Co., Inc., 1987. 
[17] P. Vázquez, M. Feixas, M. Sbert, and W. Heidrich, Automatic View Selection Using Viewpoint Entropy and its Applications to Imagebased Modelling., Comput. Graph. Forum, vol. 22, no. 4, 2003, pp. 689-700.

[18] S. Fleishman, D. Cohen-Or, and D. Lischinski, Automatic Camera Placement for Image-Based Modeling., Comput. Graph. Forum, vol. 19 , no. 2, 2000, pp. 101-110.

[19] J. Rigau, M. Feixas, and M. Sbert, New Contrast Measures for Pixel Supersampling, Springer-Verlag London Limited, 2002, pp. 439-451.

[20] P. Barral, G. Dorme, and D. Plemenos, Visual understanding of a scene by automatic movement of a camera, International Conference GraphiCon'99, 1999.

[21] T. Kamada, and S. Kawai, A simple method for computing general position in displaying three-dimensional objects, Computer Vision, Graphics, and Image Processing, vol. 41, no. 1, 1988, pp. 43-56.

[22] P. Barral, G. Dorme, and D. Plemenos, Scene understanding techniques using a virtual camera., 2000.

[23] I. Viola, M. Feixas, M. Sbert, and M.E. Groller, Importance-Driven Focus of Attention, Visualization and Computer Graphics, IEEE Transactions on, vol. 12, no. 5, 2006, pp. 933-940.

[24] L. Teng-Yok, O. Mishchenko, S. Han-Wei, and R. Crawfis, View point evaluation and streamline filtering for flow visualization, Pacific Visualization Symposium (PacificVis), 2011 IEEE, 2011, pp. 83-90.

[25] P. Vázquez, M. Feixas, M. Sbert, and A. Llobet, Realtime automatic selection of good molecular views, Computers \& Graphics, vol. 30, no. 1,2006 , pp. $98-110$.
[26] C. Ming-Yuen, Q. Huamin, C. Ka-Kei, M. Wai-Ho, and W. Yingcai, Relation-Aware Volume Exploration Pipeline, Visualization and Computer Graphics, IEEE Transactions on, vol. 14, no. 6, 2008, pp. 1683-1690.

[27] J. O'Rourke, Art gallery theorems and algorithms, Oxford University Press, Inc., 1987.

[28] C.A. Gran, P. Vázquez, and M.F. González, Way-Finder: Guided Tours Through Complex Walkthrough Models., Comput. Graph. Forum, vol. 23, no. 3, 2004, pp. 499-508.

[29] L. Wixson, Viewpoint selection for visual search, Computer Vision and Pattern Recognition, 1994. Proceedings CVPR '94., 1994 IEEE Computer Society Conference on, 1994, pp. 800-805.

[30] P. Vázquez, and M. Sbert, Automatic indoor scene exploration, 2003, pp. 13-24.

[31] P. Vázquez, On the selection of good views and its application to computer graphics., phdVaz03, Barcelona (Spain), 2003.

[32] D. Plemenos, Exploring virtual worlds: Current techniques and future issues, 2003, pp. 5-10.

[33] D. Sokolov, D. Plemenos, and K. Tamine, Methods and data structures for virtual world exploration, The Visual Computer, vol. 22, no. 7, 2006, pp. 506-516.

[34] M. Eusuff, K. Lansey, and F. Pasha, Shuffled frog-leaping algorithm: a memetic meta-heuristic for discrete optimization, Engineering Optimization, vol. 38, no. 2, 2006, pp. 129-154. 